\title{
Limitations of red noise in analysing Dansgaard-Oeschger events
}

\author{
H. Braun ${ }^{1,2}$, P. Ditlevsen ${ }^{1}$, J. Kurths ${ }^{3,4}$, and M. Mudelsee ${ }^{5}$ \\ ${ }^{1}$ Centre for Ice and Climate, Niels Bohr Institute, University of Copenhagen, Juliane Maries Vej 30, \\ 2100 Copenhagen, Denmark \\ ${ }^{2}$ Heidelberg Academy of Sciences and Humanities, Im Neuenheimer Feld 229, 69120 Heidelberg, Germany \\ ${ }^{3}$ Institute of Physics, Humboldt University Berlin, Newtonstraße 15, 12489 Berlin, Germany \\ ${ }^{4}$ Potsdam Institute for Climate Impact Research, P.O. Box 601203, 14412 Potsdam, Germany \\ ${ }^{5}$ Climate Risk Analysis, Schneiderberg 26, 30167 Hannover, Germany
}

Received: 16 June 2009 - Published in Clim. Past Discuss.: 3 July 2009

Revised: 6 January 2010 - Accepted: 10 February 2010 - Published: 19 February 2010

\begin{abstract}
During the last glacial period, climate records from the North Atlantic region exhibit a pronounced spectral component corresponding to a period of about 1470 years, which has attracted much attention. This spectral peak is closely related to the recurrence pattern of DansgaardOeschger (DO) events. In previous studies a red noise random process, more precisely a first-order autoregressive (AR1) process, was used to evaluate the statistical significance of this peak, with a reported significance of more than $99 \%$. Here we use a simple mechanistic two-state model of DO events, which itself was derived from a much more sophisticated ocean-atmosphere model of intermediate complexity, to numerically evaluate the spectral properties of random (i.e., solely noise-driven) events. This way we find that the power spectral density of random DO events differs fundamentally from a simple red noise random process. These results question the applicability of linear spectral analysis for estimating the statistical significance of highly non-linear processes such as DO events. More precisely, to enhance our scientific understanding about the trigger of DO events, we must not consider simple "straw men" as, for example, the AR1 random process, but rather test against realistic alternative descriptions.
\end{abstract}

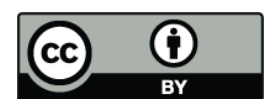

Correspondence to: H. Braun (holger.braun@iup.uni-heidelberg.de)

\section{Introduction}

Many climate records from the North Atlantic region exhibit an outstanding spectral peak corresponding to a period of about 1470 years during the last glacial period (Grootes and Stuiver, 1997; Schulz 2002). This spectral component, which has attracted much interest recently, is apparently nonstationary and particularly pronounced in the GISP2 ice core record during the time interval between 31000 and 36000 years before present (Schulz, 2002). To estimate the statistically significance of this 1470-year spectral peak, a firstorder autoregressive (AR1) random process was used in earlier studies (Schulz, 2002), which in the following we take as synonymous with a red noise random process, whose power spectral density by definition decreases monotonically with increasing frequency (Gilman et al., 1963). The nonnormalised power spectral density distribution $\operatorname{PSD}(f)$ of a discrete time series of an AR1 process (with spacing unity and innovation standard deviation $\sigma$ ) is given by the expression:

$\operatorname{PSD}(\mathrm{f})=2 \cdot \sigma^{2} /\left[1-2 a \cdot \cos (2 \pi f)+a^{2}\right]$

where $a$ is the autocorrelation parameter and $f$ denotes the frequency (Priestley, 1981). This approach resulted in a reported significance of more than $99 \%$ (Schulz, 2002). More precisely, the null hypothesis in that study was that the GISP2 stable isotope $\left(\delta^{18} \mathrm{O}\right)$ time series is generated by an AR1 random process. By means of a Monte-Carlo based numerical method, Schulz (2002) found that the magnitude of the 1470year spectral peak is too large to be generated by an AR1 random process, at a confidence level of $99 \%$. Based on this finding, Schulz (2002) regarded this spectral peak as being

Published by Copernicus Publications on behalf of the European Geosciences Union. 


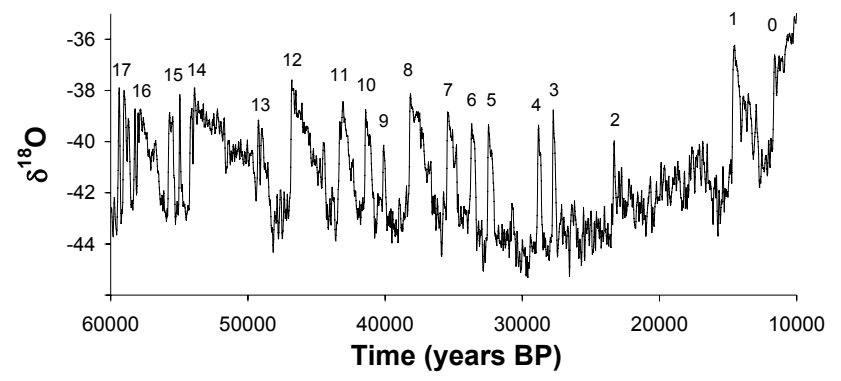

Fig. 1. Dansgaard-Oeschger (DO) events $0-17$ as seen in the NGRIP $\delta^{18} \mathrm{O}$ ice core record from Greenland during the time interval between 10000 to 60000 years before present (GICC05 time scale). A five point running mean was applied to remove the highest frequency oscillations. The accumulated error of the time scale during the considered time interval is on the order of $2 \%$ (estimated $1 \sigma$ accumulated error; Svensson et al., 2008).

"statistically significant at the $99 \%$ level". Thus, under the assumption that an AR1 random process is an appropriate null hypothesis, the 1470-year spectral peak is significant at the $99 \%$ (and also at a lower) significance level. Here we use a mechanistic model, as described in section 2 and 3, to investigate if an AR1 process constitutes a realistic null hypothesis for testing the statistical significance of this 1470year spectral peak. We explicitly stress that here it is not our intention to actually perform such a test of the significance. We further note that Schulz (2002) questioned the statistical significance of the 1470-year spectral peak, owing to its non-stationary character, and presented another method to test this apparent regularity.

In the time domain the 1470-year spectral peak is closely linked with the occurrence of Dansgaard-Oeschger (DO) events (Schulz, 2002), cf. Fig. 1. These events show some tendency to recur in near-multiples of about 1,470 years during the last glacial period (Alley, 2001; Schulz, 2002; Rahmstorf, 2003). The statistical significance of this tendency, however, is still a matter of debate (Ditlevsen et al., 2007). The standard interpretation is that DO events represent regime shifts between two different modes of the ocean/atmosphere system (Dansgaard et al., 1982; Oeschger et al., 1984; Broecker et al. 1985; Sarnthein et al., 1994; Alley and Clark, 1999), as has been concluded from climate records (e.g. Steffensen et al., 2008) and ocean-atmosphere models (e.g. Ganopolski and Rahmstorf, 2001). Transitions between both modes apparently happened very quickly, that is, on the annual to decadal time scale (Taylor et al., 1997; Severinghaus and Brook, 1999; Steffensen et al., 2008), which is commonly regarded as observational support for the existence of threshold-crossing processes during DO events (Alley et al., 2003; Steffensen et al., 2008), that is, a "tipping point" (Lenton et al., 2008). Such a threshold could be provided for example by the process of buoyancy deep convection in the ocean (“deep water formation"), which occurs when surface water gets denser than the deeper ocean water (Ganopolski and Rahmstorf, 2001). From a theoretical point of view, DO events could thus be regarded as repeated oscillations in a system with two possible states of operation and with a threshold (Braun et al., 2007).

In dynamical system theory it is well known that highly non-linear systems, for example systems with thresholds, can respond at a preferred time scale, the stochastic time scale, even when driven by a random input (i.e., "noise") only (Pikovsky and Kurths, 1997; Gammaitoni et al., 1998). The stochastic time scale corresponds to the average spacing between successive noise-induced events and is closely related with the magnitude of the noise in the forcing. In other words, noise-induced DO events are expected to evolve on a very distinctive time scale, more precisely on the millennial to multi-millennial scale (Ganopolski and Rahmstorf 2002; Braun et al., 2007). The red noise random process, in contrast, describes the spectral properties of a linear, noisedriven system with damping. In contrast to a system with a threshold, such a system responds on all time scales when driven by noise, with maximum variance on the very long time scale and decreasing variance on shorter time scales. In other words, the red noise random process might not be applicable to estimate the spectral properties of random DO events and more sophisticated approaches might be needed, based for example on Monte-Carlo simulations with models that are able to mimic the dynamics of DO events.

\section{A simple two-state model of DO events}

In this paper we use a very simple mechanistic two-state model for the dynamics of DO events (Fig. 2) to estimate the spectral properties of random (i.e., solely noise-induced) DO events. Our model is identical to the one described and used in the publications of Braun et al. (2007) and Braun et al. (2008). A comprehensive description of this model was presented in the work of Braun et al. (2007), including a detailed discussion of its physical motivation, its applicability and its limitations. This model has been derived from the dynamical principles of DO events as simulated with a much more sophisticated ocean-atmosphere model of intermediate complexity (CLIMBER-2), which itself is too slow for extensive statistical analyses. The ability of the simple two-state model to reproduce the waiting time statistics of the events in that ocean-atmosphere model was already demonstrated in a few simple forcing scenarios (Braun et al., 2007).

The dynamics of DO events in the simple two-state model is depicted in Fig. 2. It is assumed that the events represent transitions between two states of operation ("stadial"=cold state, "interstadial"=warm state) in a system with a threshold. Transitions between these states occur when a given forcing $f$ (in freshwater flux units, that is, in $\mathrm{mSv} ; 1 \mathrm{mSv}=1$ milliSverdrup $=10^{3} \mathrm{~m}^{3} / \mathrm{s}$ ) crosses a certain threshold function $T$. More precisely, a switch from the cold state to the warm 
Table 1. Parameters of the simple two-state model. Values of $A_{0}$, $A_{1}, B_{0}$ and $B_{1}$ are im $\mathrm{mSv}\left(1 \mathrm{mSv}=1\right.$ milli-Sverdrup $\left.=10^{3} \mathrm{~m}^{3} / \mathrm{s}\right)$, that it is freshwater flux units, since the model was originally designed to mimic the response of an ocean-atmosphere model to a given freshwater anomaly in the northern North Atlantic. Note that these parameter values are identical to the values used in the original version of the two-state model, cf. supplementary material in the publication of Braun et al. (2005). For these values it was shown that the two-state model is able to mimic the dynamical principles of DO events as simulated with the model CLIMBER-2, a much more comprehensive ocean-atmosphere model of "intermediate complexity" (Braun et al., 2007). The tuning procedure of the model parameters is described in detail in the author comment in the CPD discussion forum (Braun et al., 2009b).

\begin{tabular}{lr}
\hline Parameter & Value \\
\hline$A_{0}$ & $-27 \mathrm{mSv}$ \\
$A_{1}$ & $27 \mathrm{mSv}$ \\
$B_{0}$ & $-9.7 \mathrm{mSv}$ \\
$B_{1}$ & $11.2 \mathrm{mSv}$ \\
$\tau_{0}$ & 1200 years \\
$\tau_{1}$ & 800 years \\
\hline
\end{tabular}

state happens when $f<T$. The opposite switch occurs when $f>T$. During the switches a discontinuity in the threshold function is assumed, that is, $T$ overshoots and afterwards approaches its respective equilibrium value following a relaxation process with a millennial time scale (Fig. 2). We note that in the model, the onset of a DO event is represented by the transition from stadial ("cold") conditions to interstadial ("warm") ones, at time $t_{0}$ in Fig. 2. The opposite transition (at time $t_{1}$ ) represents the termination of an event. Thus, the expression "Dansgaard-Oeschger event" is used for the time interval between these two transitions, that is, for the entire duration of the "warm" state, following standard paleoclimatic nomenclature.

Note that the dynamical principles and the transition rules in the two-state model are a first order approximation of the dynamics of DO events in the ocean-atmosphere model CLIMBER-2 (Petoukhov et al., 2000; Ganopolski and Rahmstorf, 2001). In that model DO events also represent threshold-crossing events in a system with two possible states of operation (corresponding to two fundamentally different modes of buoyancy deep convection in the northern North Atlantic) and with an overshooting in the stability of the system during these shifts (Ganopolski and Rahmstorf, 2001; Braun et al., 2007). Analogous to the simple two-state model, switches from the stadial mode to the interstadial one are triggered by sufficiently large negative forcing anomalies (more precisely, by a reduction in the surface freshwater flux to the northern North Atlantic that exceeds a certain threshold value), whereas the opposite shifts are triggered by sufficiently large positive forcing anomalies (that is, by an
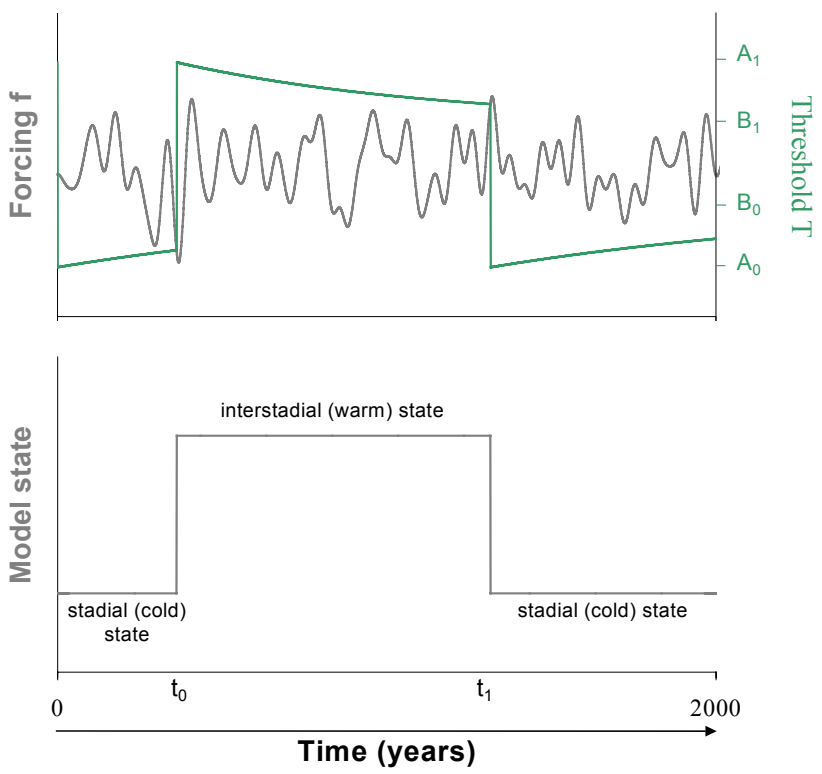

Fig. 2. Dynamics of DO events in the two-state model. Top: Forcing (grey) and threshold function (green). Bottom: Model state. A switch from the stadial to the interstadial state is triggered when the forcing falls below the threshold function (at time $t_{0}$ in the figure). During this switch, which is interpreted as the beginning of a DO event in the model, the threshold function takes a non-equilibrium value $\left(A_{1}\right)$ and afterwards approaches its new equilibrium $B_{1}$ following a millennial scale relaxation process with relaxation time $\tau_{1}$. The opposite switch, which terminates a DO event in the model, takes place when the forcing exceeds the threshold function (at time $t_{1}$ in the figure). Again, the threshold function takes a nonequilibrium value $\left(A_{0}\right)$ and approaches its new equilibrium value $B_{0}$ following another millennial scale relaxation process with relaxation time $\tau_{0}$. All model parameters are given in Table 1 .

increase in the freshwater flux that exceeds a certain threshold value). The simple two-state model has six independent parameters (Table 1), which have also been estimated from the ocean-atmosphere model CLIMBER-2, as demonstrated in the supporting online material in the publications of Braun et al. (2005) and (2007).

To illustrate the agreement between the simple two-state model and the ocean-atmosphere model CLIMBER-2 we here present one example (Fig. 3). A detailed comparison, which also includes some more examples, can be found in the supplementary material of the publication of Braun et al. (2007). Figure 3 shows the response of both models to a periodic, bi-sinusoidal forcing in cycles of about 210 and 86.5 years, respectively. The amplitude of the forcing is chosen to be supra-threshold, because a sub-threshold forcing is not able to trigger repeated DO events in either of the models. As can be seen from Fig. 3, to a reasonable approximation the conceptual model is able to reproduce several aspects of the DO events as simulated with the oceanatmosphere model, for example the timing and the duration 


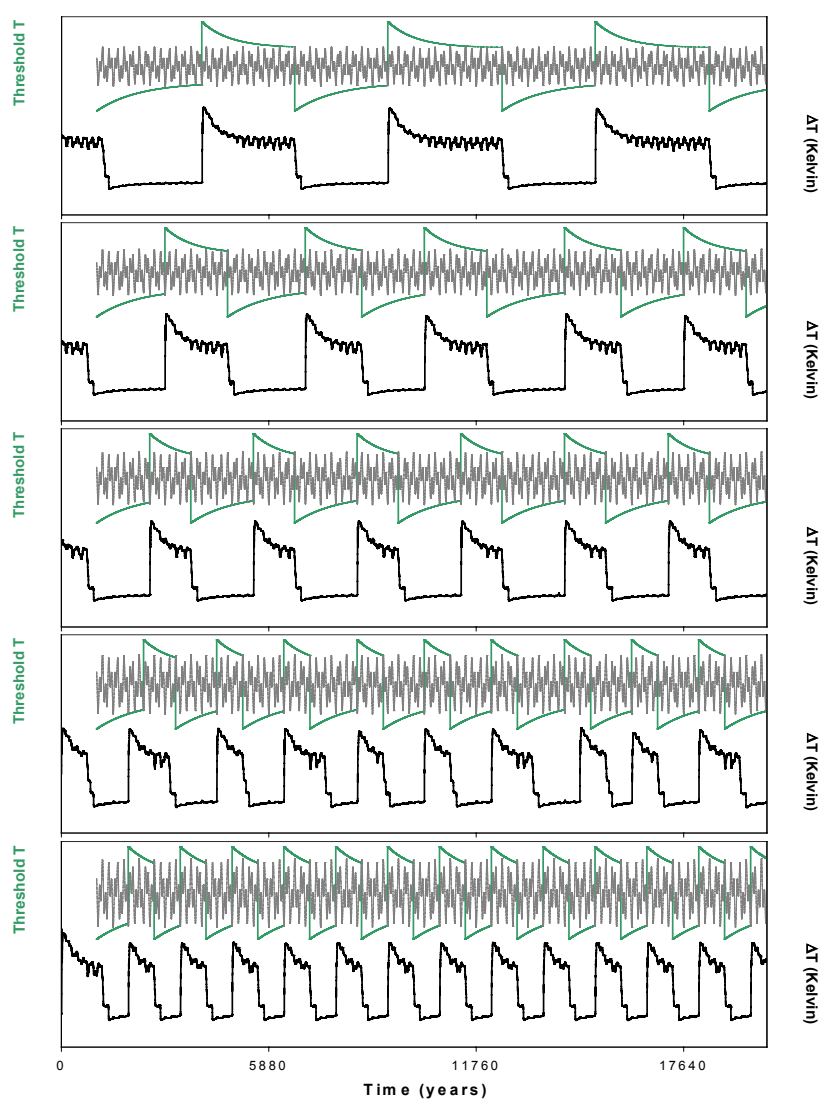

Fig. 3. Comparision between the simple two-state model and the ocean-atmosphere model of intermediate complexity, CLIMBER-2. The figure shows the output of the simple two state model (green) and of the ocean-atmosphere model (black), in response to a simple periodic forcing function consisting of two century-scale spectral components of equal amplitude (grey) and periods of 1470/7 and $1470 / 17$ years, inspired by the periods of the Gleissberg ( $~ 88$ years) and DeVries/Suess ( $\sim 208$ years) solar cycles. Note that the forcing amplitude increases from the top to the bottom (from $6 \mathrm{mSv}$ in the top panel to $10 \mathrm{mSv}$ in the bottom panel) whereas the waiting time between successive events decreases. In particular, the two-state model reproduces the onset and the termination of the events in the ocean-atmosphere model fairly well. A more detailed comparison between both models exists in the work of Braun et al. (2007) and in the supplementary material of that publication.

of the events, the overshooting during the transitions between both model states, the subsequent millennial relaxation process and the decrease of the inter-event waiting times when the forcing amplitude increases. In this sense, the conceptual model apparently has the ability to mimic the main principles that govern the dynamics of DO events in the oceanatmosphere model CLIMBER-2. Note that the response of the conceptual model is given by the time evolution of the threshold function $T$, whereas in the CLIMBER-2 model the response is given by the surface air temperature anomalies in the North Atlantic region during the simulated DO-like events. When comparing these anomalies with the stable isotope time series from the ice cores (Fig. 1), it must be kept in mind that uncertainties exist for example due to the conversion between $\delta^{18} \mathrm{O}$ and North Atlantic/Greenland temperature (Masson-Delmotte et al., 2006).

\section{Spectral properties of random DO events}

In the following we use this simple two-state model to evaluate the spectral properties of random DO events. This is done in the following: We force the model by a random, Gaussian-distributed input with white-noise power signature within a certain spectral interval. Let $\sigma$ be the standard deviation of the noise. The cut-off frequency of the noise is $1 / 50$ years $^{-1}$. In other words, for spectral components with frequency higher than $1 / 50$ years $^{-1}$ the amplitude of the noise-term is zero. For lower frequencies a uniform amplitude distribution ("white noise") is used. The cut-off is applied following the publication of Braun et al. (2007) to account for the fact that the simple two-state model shows an unrealistic large sensitivity to decadal-scale or faster forcing. Note that the magnitude and the spectral composition of the noise in the freshwater flux to the North Atlantic is of course unknown during the last Glacial, so that it is inevitable to make some simplified assumptions concerning the structure of the noise term. In particular, it is impossible to infer the high-frequency tail of the power spectral density distribution of the noise from the ice core data, since these have a time resolution of only one data point per 20 years. We thus do not see any feasible alternative to using a highly simplified noise term. Finally we calculate the spectral properties of the model response (i.e. of the threshold function $T$, which resembles the saw-tooth shape of DO events) to the forcing, following standard Fourier spectral analysis. Our focus is the question whether or not an AR1 process is a realistic assumption for the power spectral density distribution of noisedriven DO events.

Figure 4 shows the response of the two-state model for three forcing scenarios with different noise magnitude $\sigma$ As expected, the average spacing between successive events decreases with increasing magnitude of the noise, since a larger forcing can trigger more threshold crossings. The model can show surprisingly regular oscillations on the millennial time scale even when driven by noise only, as depicted in Fig. 4. In the spectral domain the output of the model can thus show outstanding spectral peaks on the millennial time scale, which are clearly inconsistent with red noise (i.e., with a first-order autoregressive [AR1] process, cf. Fig. 4, based on which the statistical significance of the 1470-year spectral peak in the GISP2 stable isotope time series has been inferred; Schulz, 2002), despite the fact that the model is driven by a random input. From Figs. 4 and 5 it is also evident that the events in the output of the system occur on a characteristic time scale, which is the millennial to multi-millennial 

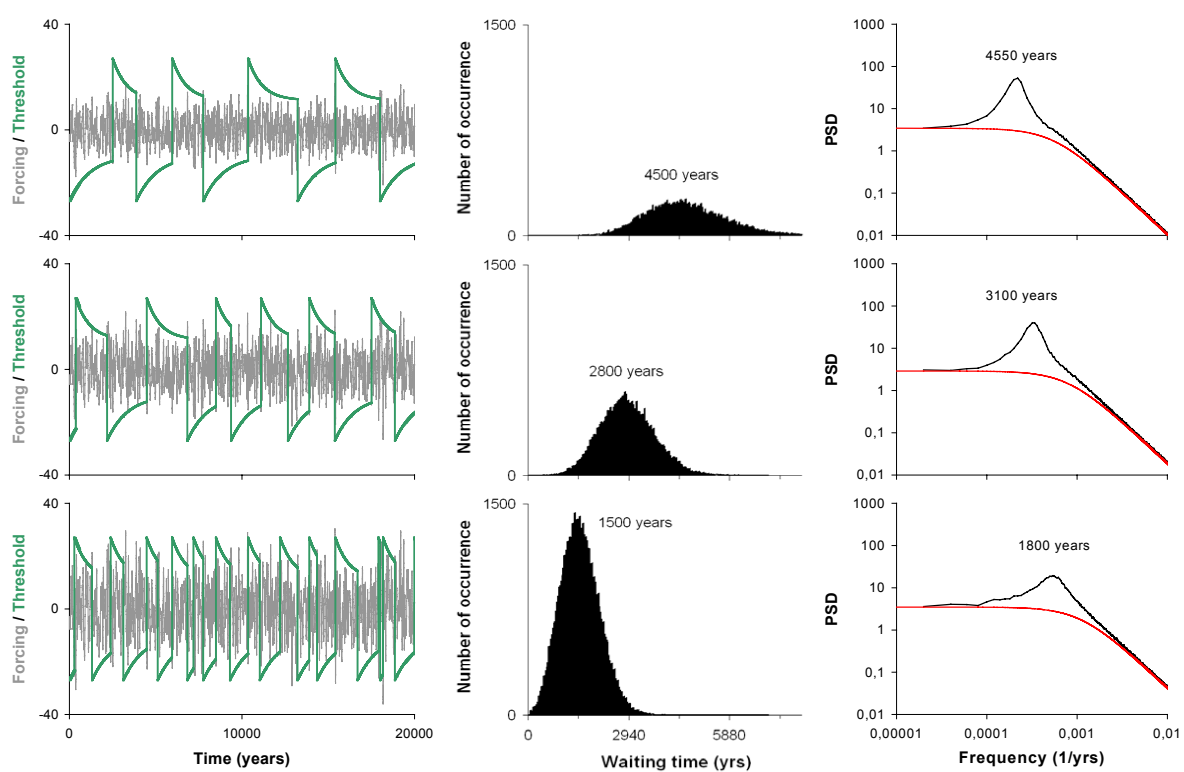

Fig. 4. Output of the two-state model. The figure shows the input (grey) and the response of the model (green). The magnitude of the noise is 5.5 (top), 7 (middle) and 9.5 (bottom). Units are $\mathrm{mSv}=$ milli-Sverdrup $=10^{3} \mathrm{~m}^{3} / \mathrm{s}$. The green curves show the model response in the time domain, that is, the time evolution of the threshold function (left). The waiting time distribution between successive events is depicted in the middle column. The distributions are obtained from 100000000 -year runs with the simple two-state model. The right column shows the power spectral density distribution of the simulated events. The power spectral density distributions are obtained from 50000-year runs, averaged over 1000 different realisations with the same noise magnitude. The red curve represents a theoretical red noise (AR1) random process, cf. Eq. (1), based on which the statistical significance of the 1470 -year spectral peak in the GISP2 $\delta^{18} \mathrm{O}$ ice core record has been inferred (Schulz, 2002). Note that the simulated power spectral density distributions show a pronounced hump at the millennial time scale, with a magnitude that is on the order of 10 times larger than expected from a red noise process.

scale. As expected, this time scale is determined by the magnitude $\sigma$ of the noise (cf. Fig. 5). The fact that the model output occurs on a distinct time scale is also evident from the power spectral density distribution of the simulated events (right column in Fig. 4), which exhibits a prominent maximum on the millennial time scale, corresponding approximately to the inverse of the average spacing between successive DO events in the simulation (Figs. 4 and 5). Leaving this maximum aside, the power spectral density distribution of the simulated events fits an AR1 random process fairly well. However, the maximum in the simulated power spectral distribution is considerably larger than expected from that process (Fig. 4).

We explicitly tested that these results are not unique to our specific forcing scenario, but are also obtained in the case of other random forcings, with somewhat different spectral composition and cut-off frequency (compare for example Fig. R2 in the author comment in the CPD discussion forum; Braun et al., 2009a, c). Moreover, we also tested that our findings are not unique to our simple conceptual model of DO events, but are likewise obtained with the oceanatmosphere model CLIMBER-2, from which the conceptual model was constructed (Fig. 6). In contrast, within the framework of this study it is not possible to discuss the stability of our findings with respect to parameter changes in the sim- ple conceptual model or even the ocean-atmosphere model. Such changes might result for example from longer term (i.e., orbital scale) climatic background variability. Similarly, it is not within the scope of our work to investigate how the statistical properties of DO events in the ice core records are influenced by observational noise, which is absent in our model simulations. We would further like to stress that the main assumption in our approach is that we consider DO events as highly non-linear processes, that is, as regime switches in a system with a threshold. In contrast, an AR1 process, which we find to be inapplicable as a null hypothesis for random DO events, represents a noise-driven linear process. Consequently, a possible test of our key assumption could be to investigate the ice core $\delta^{18} \mathrm{O}$ time series for indications of non-linearity (Kantz and Schreiber, 1997).

Our results suggest that red noise is not applicable to estimate the statistical significance of the 1470-year spectral peak of DO events, since even in our simple model the use of red noise would typically lead to a strong overestimation of the significance of spectral components on the millennial time scale. In other words, based on our simplified forcing scenarios it is not possible the exclude the idea that the pronounced glacial 1470-year peak of DO events is just random and that the reported $99 \%$ significance of the peak in the GISP2 ice core record results solely from the inappropriate 

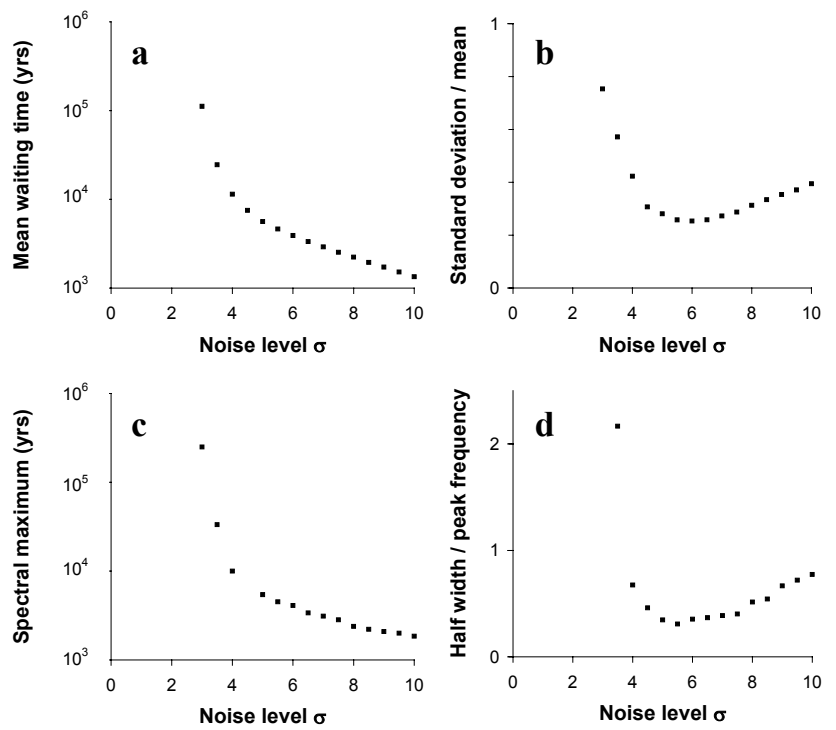

Fig. 5. Time scale and regularity of the model output as a function of the noise level. The figure shows (a) the mean waiting time between successive events in the two-state model as a function of the noise magnitude, (b) the ratio between the standard deviation of the inter-event spacing and their mean waiting time, which constitutes a measure for the regularity of the events, (c) the period of the leading spectral component (defined by the maximum of the spectral power) in the model output as a function of the noise magnitude, and (d) the ratio between the half-width of the spectral hump and its leading frequency, which constitutes another measure of regularity. Units of the noise are $\mathrm{mSv}=$ milli-Sverdrup $=10^{3} \mathrm{~m}^{3} / \mathrm{s}$. No events occur for a noise level of $2 \mathrm{mSv}$ or smaller. Note that the curves in (b) and (d) exhibit a minimum (i.e., maximum regularity) for an intermediate noise level, which indicates the existence of a solely noise-induced resonance in the model output.

use of linear methods for analysing highly non-linear processes such as DO events. We further note from Fig. 5b and $\mathrm{d}$ that in our model simulations a non-zero noise level exists, for which the regularity of the simulated events is maximised. This indicates the existence of a solely noise-induced resonance in the model output, which is characteristic for many excitable systems (Pikovsky and Kurths, 1997). This mechanism could provide a possible explanation for the recurrence pattern of the observed DO events (Ganopolski and Rahmstorf, 2002; Timmermann et al., 2003; Ditlevsen et al., 2005) or other climatic phenomena (Pelletier, 2003). Alternative hypotheses have also been proposed, involving for example synchronisation resulting from the combined effect of solar variability and noise (Braun et al., 2008).

As a final comment it should be stressed that our results do not in any way exclude the possibility that DO events exhibit characteristics in their recurrence properties which are indeed inconsistent with a random occurrence. However, this has so far not been shown in a rigorous statistical approach (Braun, 2009; Ditlevsen and Ditlevsen, 2009) and thus needs
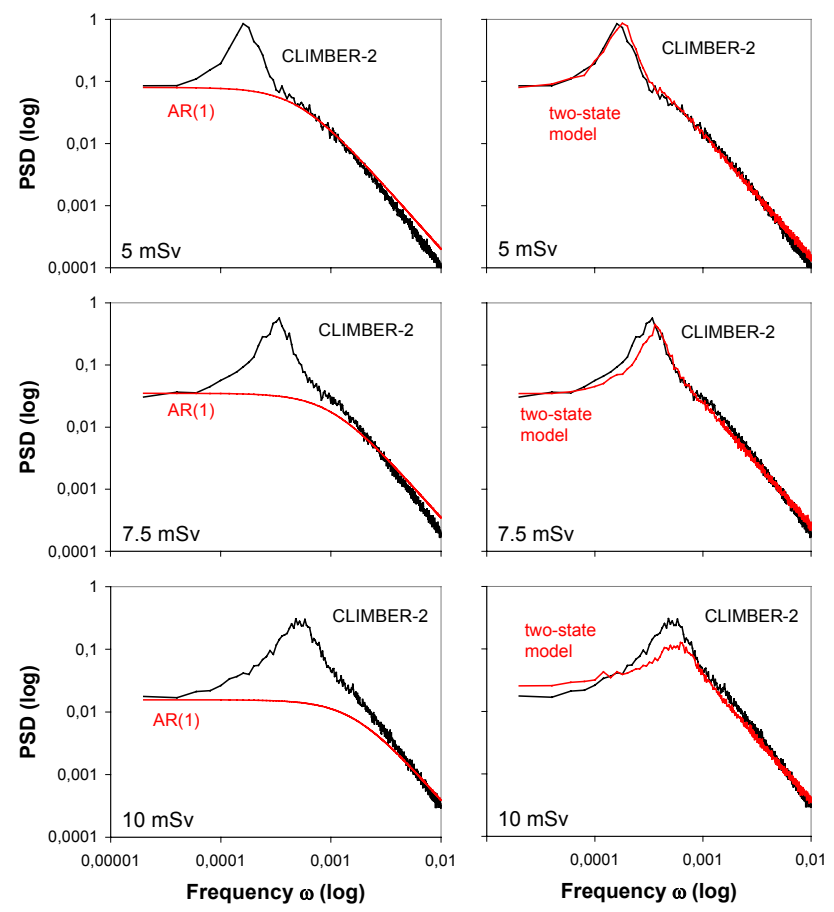

Fig. 6. Comparison of the power spectral density (PSD) distributions as obtained from the three noise-driven processes. The black curve shows the distribution as simulated with the oceanatmosphere model CLIMBER-2 over a Monte-Carlo ensemble of 100 members, each representing a 50000 -year long time series of random (i.e., noise-driven) DO events. The standard deviation of the freshwater noise in the model input is $5 \mathrm{mSv}$ (top), $7.5 \mathrm{mSv}$ (middle) and $10 \mathrm{mSv}$ (bottom). $1 \mathrm{mSv}=1$ milli-Sverdrup $=10^{3} \mathrm{~m}^{3} / \mathrm{s}$. The red curve shows the fitted distributions as obtained from a first-order autoregressive (AR1) random process (left) and from the two-state model (right). Note that in this comparison, we forced the ocean-atmosphere model CLIMBER-2 and the two-state model with precisely the same random input. In the model CLIMBER-2, this input is added as a surface freshwater anomaly in the latitudinal belt between 50 and $70^{\circ} \mathrm{N}$ in the North Atlantic. In the two-state model, this input is implemented as the forcing function $f$. To optimise the agreement between both models, we only allow for a linear scaling of the output of the two-state model, in order to account for the fact that this output is in freshwater flux units $(\mathrm{mSv})$, whereas the output of the ocean-atmosphere model is in temperature units (K). We thus have only one tunable fitting parameter, that is, a simple factor of proportionality. Note that an AR1 process cannot reproduce the existence of the pronounced millennial spectral hump in the distribution as simulated by the ocean-atmosphere model. This feature, in contrast, is reproduced by the simple two-state model, as well as the width of this "resonance hump", the position of its maximum as a function of the noise intensity, and the approximate $1 / \omega^{2}$-proportionality for high frequencies. 
to be tested in the future. We would recommend methods that allow to estimate the statistical significance without making use of linear theories, for example Monte Carlo simulations with models of DO events (Ditlevsen et al., 2007; Braun et al., 2008; Ditlevsen and Ditlevsen, 2009) or non-linear methods such as recurrence plots (Marwan and Kurths, 2002; Marwan et al., 2007, Schinkel et al., 2009).

\section{Conclusions}

In this paper it was shown that the spectral properties of highly non-linear processes such as simulated DO events can be fundamentally different from a red noise random process, even when driven by a random forcing. In this sense, red noise is no feasible null hypothesis against which to test the statistical significance of the 1470-year spectral peak of DO events, since the use of red noise could lead to a strong overinterpretation of the reported $99 \%$ significance of that 1470year peak. Future work should focus on the development of more efficient measures of regularity and on the use of more realistic random processes for Monte-Carlo based statistical analysis of DO events. Based on these methodological improvements, future analyses might be able to better address the question what triggered the remarkable DansgaardOeschger events in glacial climate.

Acknowledgements. H. B. was funded by the German Research Foundation (DFG), project number: BR 3911/1-1. J. K. was funded by SFB 555, project C1 (DFG).

Edited by: V. Masson-Delmotte

\section{References}

Alley, R. B. and Clark, P. U.: The deglaciation of the Northern Hemisphere: A global perspective, Annu. Rev. Earth Planet. Sci., 27, 149-182, 1999.

Alley, R. B., Anandakrishnan, S., and Jung, P.: Stochastic resonance in the North Atlantic, Paleoceanography, 16, 190-198, 2001.

Alley, R., Marotzke, J., Nordhaus, W., Overpeck, J., Peteet, D., Pielke Jr., R., Pierrehumbert, R., Rhines, P., Stocker, T., Talley, L. and Wallace, J.: Abrupt Climate Change, Science, 299, 20052010, 2003.

Braun, H., Christl, M., Rahmstorf, S., Ganopolski, A., Mangini, A., Kubatzki, C., Roth, K., and Kromer, B.: Possible solar origin of the 1470-year glacial climate cycle demonstrated in a coupled model, Nature, 438, 208-211, 2005.

Braun, H., Ganopolski, A., Christl, M., and Chialvo, D. R.: A simple conceptual model of abrupt glacial climate events, Nonlin. Processes Geophys., 14, 709-721, 2007,

http://www.nonlin-processes-geophys.net/14/709/2007/.

Braun, H., Ditlevsen, P. D., and Chialvo, D. R.: Solar forced Dansgaard-Oeschger events and their phase relation with solar proxies, Geophys. Res. Lett., 35, L06703, doi:10.1029/2008GL033414, 2008.
Braun, H.: Measures of periodicity for time series analysis of threshold-crossing events, Eur. Phys. J. Special Topics, 174, 3347, 2009.

Braun, H., Ditlevsen, P., Kurths, J., and Mudelsee, M.: Limitations of red noise in analysing Dansgaard-Oeschger events, Clim. Past Discuss., 5, 1803-1818, 2009a, http://www.clim-past-discuss.net/5/1803/2009/.

Braun, H.: Interactive comment on "Limitations of red noise in analysing Dansgaard-Oeschger events" by H. Braun et al., Clim. Past Discuss., 5, C834-C841, 2009b.

Braun, H.: Interactive comment on "Limitations of red noise in analysing Dansgaard-Oeschger events" by H. Braun et al., Clim. Past Discuss., 5, C861-C865, 2009c.

Broecker, W. S., Peteet, D. M., and Rind, D.: Does the oceanatmosphere system have more than one stable mode of operation?, Nature, 315, 21-26, 1985.

Dansgaard, W., Clausen, H. B., Gundestrup, N., Hammer, C. U., Johnsen, S. F., Kristinsdottir, P. M., and Reeh, N.: A New Greenland Deep Ice Core, Science, 218, 1273-77, 1982.

Ditlevsen, P. D., Kristensen, M. S., and Andersen, K. K.: The recurrence time of Dansgaard-Oeschger events and possible causes, J. Climate, 18, 2594-2603, 2005.

Ditlevsen, P. D., Andersen, K. K., and Svensson, A.: The DOclimate events are probably noise induced: statistical investigation of the claimed 1470 years cycle, Clim. Past, 3, 129-134, 2007 , http://www.clim-past.net/3/129/2007/.

Ditlevsen, P. D. and Ditlevsen, O. D.: On the Stochastic Nature of the Rapid Climate Shifts during the Last Ice Age, J. Clim., 22, 446-457, 2009.

Gammaitoni, L., Hänggi, P., Jung, P., and Marchesoni, F.: Stochastic resonance, Review of Modern Physics, 70(1), 223-287, doi:10.1103/RevModPhys.70.223, 1998.

Ganopolski, A. and Rahmstorf, S.: Simulation of rapid glacial climate changes in a coupled climate model, Nature, 409, 153-158, 2001.

Ganopolski, A. and Rahmstorf, S.: Abrupt glacial climate changes due to stochastic resonance, Phys. Rev. Lett., 88(3), 038501, doi:10.1103/PhysRevLett.88.038501, 2002.

Gilman, D., Fuglister, F., and Mitchell, J.: On the power spectrum of red noise, J. Atmos. Sci., 20(2), 182-184, 1963.

Grootes, P. M. and Stuiver, M.: Oxygen 18/16 variability in Greenland snow and ice with $10^{3}$ to $10^{5}$-year time resolution, J. Geophys. Res., 102(C12), 26455-26470, 1997.

Kantz, H. and Schreiber, T.: Nonlinear time series analysis. Cambridge Nonlinear Science Series No. 7, Cambridge University Press, Cambridge UK, 1997.

Lenton, T. M., Held, H., Kriegler, E., Hall, J. W., Lucht, W., Rahmstorf, S., and Schellnhuber, H. J.: Tipping elements in the Earth's climate system, Proc. Natl. Acad. Sci. USA, 105, 1786-1793, 2008.

Masson-Delmotte, V., Dreyfus, G., Braconnot, P., Johnsen, S., Jouzel, J., Kageyama, M., Landais, A., Loutre, M.-F., Nouet, J., Parrenin, F., Raynaud, D., Stenni, B., and Tuenter, E.: Past temperature reconstructions from deep ice cores: relevance for future climate change, Clim. Past, 2, 145-165, 2006, http://www.clim-past.net/2/145/2006/.

Marwan, N. and Kurths, J.: Nonlinear analysis of bivariate data with cross recurrence plots, Phys. Lett. A., 302, 299-307, 2002. 
Marwan, N., Romano, M. C., Thiel, M., and Kurths, J.: Recurrence plots for the analysis of complex systems, Physics Reports, 438, 5-6, doi:10.1016/j.physrep.2006.11.001, 2007.

Oeschger, H., Beer, J., Siegenthaler, U., Stauffer, B., Dansgaard, W., Langway, C. C.: Late glacial climate history from ice cores, in: Climate Processes and Climate Sensitivity, Geophys. Monogr. Ser., vol. 5, edited by: Hansen, J. E. and Takahashi, T., 299-306, AGU, Washington, D.C., 1984.

Pelletier, J. D.: Coherence resonance and ice ages, J. Geophys. Res., 108(D20), 4645, doi:10.1029/2002JD003120, 2003.

Petoukhov, V., Ganopolski, A., Brovkin, V., Claussen, M., Eliseev, A., Kubatzki, C., and Rahmstorf, S.: CLIMBER-2: A climate system model of intermediate complexity. Part I: Model description and performance for present climate, Clim. Dynam., 16, 117, 2000.

Pikovsky, A. S. and Kurths, J.: Coherence resonance in a noisedriven excitable system, Phys. Rev. Lett., 78, 775-778, 1997.

Priestley, M. B.: Spectral Analysis and Time Series (Volumes $1 \&$ 2), Academic Press, London, 1981

Rahmstorf, S.: Timing of abrupt climate change: a precise clock, Geophys. Res. Lett., 30(10), 1510, doi:10.1029/2003GL017115, 2003.

Sarnthein, M., Winn, K., Jung, S. J. A., Duplessy, J. C., Labeyrie, L., Erlenkeuser, H., and Ganssen, G.: Changes in East Atlantic Deepwater Circulation over the Last 30000 Years: Eight Time Slice Reconstructions, Paleoceanography, 9, 209-267, 1994.

Schinkel, S., Marwan, N., Dimigen, O., and Kurths, J.: Confidence bounds of recurrence-based complexity measures, Phys. Lett. A., 26, 2245-2250, 2009.

Schulz, M.: On the 1470-year pacing of DansgaardOeschger warm events, Paleoceanography, 17(2), 1014, doi:10.1029/2000PA000571, 2002.
Severinghaus, J. P. and Brook, E.: Abrupt Climate Change at the End of the Last Glacial Period Inferred from Trapped Air in Polar Ice, Science, 286, 930-934, 1999.

Steffensen, J. P., Andersen, K. K., Bigler, M., Clausen, H. B., Dahl-Jensen, D., Fischer, H., Goto-Azuma, K., Hansson, M., Johnsen, S. J., Jouzel, J., Masson-Delmotte, V., Popp, T., Rasmussen, S. O., Rothlisberger, R., Ruth, U., Stauffer, B., SiggaardAndersen, M.-L., Sveinbjörnsdottir, A. E., Svensson, A., and White, J. W. C.: High-Resolution Greenland Ice Core Data Show Abrupt Climate Change Happens in Few Years. Science, doi:10.1126/science.1157707, 2008; Published Online 19 June, Science Express, 2008.

Svensson, A., Andersen, K. K., Bigler, M., Clausen, H. B., DahlJensen, D., Davies, S. M., Johnsen, S. J., Muscheler, R., Parrenin, F., Rasmussen, S. O., Rthlisberger, R., Seierstad, I., Steffensen, J. P., and Vinther, B. M.: A 60000 year Greenland stratigraphic ice core chronology, Clim. Past, 4, 47-57, 2008, http://www.clim-past.net/4/47/2008/.

Taylor, K. C., Mayewski, P. A., Alley, R. B., Brook, E. J., Gow, A. J., Grootes, P. M., Meese, D. A., Saltzman, E. S., Severinghaus, J. P., Twickler, E. S., White, J. W. C., Whitlow, S., and Zielinski, G. A.: The Holocene-Younger Dryas Transition Recorded at Summit, Greenland, Science, 278, 825-827, 1997.

Timmermann, A., Gildor, H., Schulz, M., and Tziperman, E.: Coherent Resonant Millennial-Scale Climate Oscillations Triggered by Massive Meltwater Pulses, J. Climate, 16, 2569-2585, 2003. 